\title{
Optical biosensor based on arrays of waveguide microcantilevers
}

\author{
Kirill Zinoviev ${ }^{\text {a) }}$, Jose Antonio Plaza, Victor Cadarso, Carlos Dominguez, and \\ Laura M. Lechuga \\ Centro Nacional de Microelectrónica, CSIC, \\ campus UAB, Cerdanyola del Valles, 08193, Spain \\ tlf: +34935947700 \\ a) The author to whom the correspondence should be addressed electronic mail is Kirill.Zinoviev@cnm.es
}

\begin{abstract}
Deflection of a microcantilever caused by any kind of biochemical reaction occurring on its surface can be detected with subangstrom resolution if an appropriate detection technique is exploited. This kind of transducers has become widely used in biological research since a few years ago. Usually, for the readout of the nanomechanical response of the micro beams to bio-specific interactions, a technique similar to one used in the atomic force microscopy is employed. The optical read-out method has some disadvantages, such as low degree of integration and difficulties in work with arrays of cantilevers. In the technique presented in this work the cantilever itself is an optical waveguide butt-coupled with another one. The device is fabricated as an array of 20 waveguide cantilever channels which allows for higher integration level. The analysis of the capabilities of the device, the problems associated with the design and the fabrication of the device, the choice of the material and the technology for the fabrication of very flat cantilevers have been successfully addressed. The characterisation of the device was done, showing that the resolution of the device is comparable with the one using the optical lever read-out. Results of the simulations and experimental data on the optical cantilevers coated with an absorbent material will be presented. The choice of the appropriate thickness of the absorbent material on the cantilever surface allows for acceptable losses, for single mode behaviour and adjustment of the initial displacement of the cantilever.
\end{abstract}

Keywords: microcantilevers, optical waveguides, biosensing.

\section{INTRODUCTION}

Over the last two decades, advances in micromachining technologies have promoted the development of micromechanical sensors and, in particularly, the sensors based on microcantilevers. The microcantilever sensors have become a subject of extensive research efforts and have started to became widely used in a number of applications, where the detection of ultra weak forces is needed. Biosensors based on microcantilevers is a promising tool for direct detection of biomolecular interactions with high accuracy [1]. Microcantilevers translate recognition of bio-molecules into nanomechanical motion. Picomolar or femtomolar levels of a great number of biological and chemical substances could be detected by the cantilevers. The sensors can be applied in areas such as environmental monitoring, industrial and food processing, healthcare, biomedical technology, and clinical analysis.

Extensive reviews about cantilever sensors can be found in the literature $[1,2,3]$. The cantilevers are miniature beams anchored at one end. The transduction of mechanical energy and nanomechanical motion are involved in the mechanism of operation of these devices. Development of the elasticity theory by S. Timoshenko [4] and experimental studies of thin films by Stoney [5] allowed to create fundamental analytical models which can be applied for simulation of the cantilevers behaviour.The 
displacement of a cantilever subject for example either to force exerted on it or to intrinsic stress generated on its surface can be measured with sub angstrom accuracy using various, methods, developed generally for scanning force microscopy needs. Further integration of the devices and necessity of using the reference channels in the experiments resulted in the development of cantilevers arrays [6-9]. While working with the arrays the question of accurate readout of the information from the cantilever transducers and integration level strongly depends on the detection technique. Among the most known methods are: optical lever [10], interferometric [11], piezoresistive [12], piezoelectric, capacitance readout, a recently demonstrated method based on integrated MOSFET transistors [13]. There also have been other methods reported [14-16].

The optical lever is characterised by high sensitivity (the typical sensitivity, defined as fractional change in the output signal per unit cantilever displacement, is about $10^{-3} \mathrm{~nm}^{-1}$ ), however, low integration level complicates the performance of the experiments in series, when arrays of the cantilevers are involved. This problem has been some how resolved using arrays of VCSELs [6-9] or by sequential scanning of the cantilever using mechanical scanning of the laser across the cantilevers [19]. Based on the same principle the devices monitoring the cantilever profiles were demonstrated: The profile of the cantilever controlled by sequential 2D scanning in the cantilevers plane [17], or simultaneous monitoring of several points along a single cantilever using an array of VCSELs [18]. There are a number of commercially available platforms based on the optical lever technique [1]. However, the system still is difficult to adjust when working with cantilevers arrays, so the method can not be considered as a highly integrated approach.

The static deflections as small as $5 \mathrm{~nm}$ were registered by integrated MOSFETs [13], which is large compared with the detection limit of a standard AFM technique, but the integration level which can be achieved makes the device attractive.

Cantilevers with piezoresistive readout [12] are highly integrated devices able to work in non transparent liquids and requiring less alignment and adjustment. Low sensitivity $\left(10^{-6} \mathrm{~nm}^{-1}\right)$ is a drawback of the piezocantilevers. Interferometric methods [11] demonstrated the best sensitivity, 6 $\mathrm{fm} / \sqrt{\mathrm{Hz}}$ (expressed in terms of the deflection noise density), but so far were not commercialised.

\section{OPTICAL WAVEGUIDE CANTILEVER (OWC)}

In almost all the methods, except when the cantilever profile real-time monitoring is realised, the information about the expected reaction is obtained by monitoring the cantilever free end displacement. This displacement can be registered also using two butt-coupled waveguides if the cantilever is one of them. In [20] we presented an analysis of sensitivity of a sensor based on the optical waveguide cantilever transducers, assuming that the shot noise of the photodetector is the major factor limiting the sensitivity of the device. The present work is focused on some aspects related with the design, fabrication technology of the sensor, performance of the metallised optical waveguide cantilevers, light coupling and chip encapsulation.

The sensor is an array of waveguide channels. Each channel contains: an input waveguide for light delivery, a cantilever, which itself acts as a waveguide, and an output waveguide, also called receptor (see Fig. 1). The cantilevers on chip were located in a common cavity. The gap between the cantilever and the output waveguide was fixed to $3 \mu \mathrm{m}$. The cantilever has low internal stress gradient and is practically flat as it was checked by confocal microscopy. Light delivered to the cantilever by the input waveguide propagates along it and then upon exiting from the cantilever is coupled into the receptor. If the optical characteristics of the system remain constant, the output light power depends on the cantilever deflection only. 


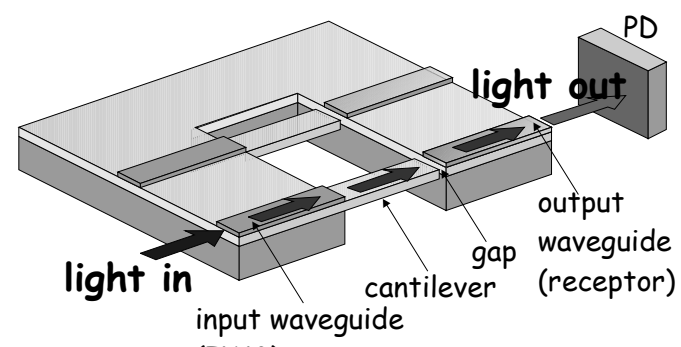

(IWG)

Fig. 1. Schematic view of the sensor.

The fabricated device, presented in Fig. 2, contains an array of 20 waveguide cantilevers. Samples with $200 \mu \mathrm{m}$ long $40 \mu \mathrm{m}$ wide and $0.6 \mu \mathrm{m}$ thick cantilever rectangular beams were produced. According to the simulations, the cantilevers supported up to 3 guiding modes in air and 2 modes in water. Silicon nitride input and output waveguides were $140 \mathrm{~nm}$ thick.

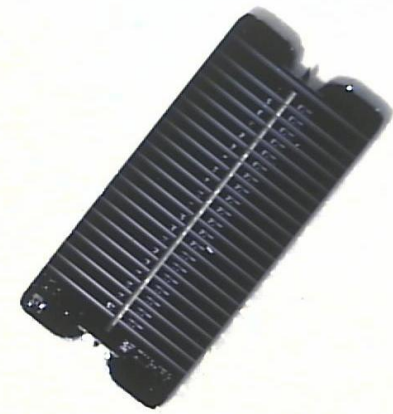

a)

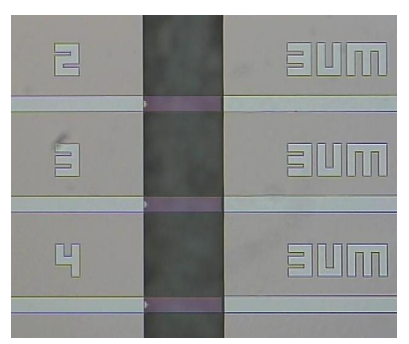

b)

Fig. 2. a) A photo of the OWC chip and b) a magnified photo of the cantilevers.

In order to characterise the sensor, an experimental setup with the both optical lever and the proposed technique was assembled (Fig. 3). Light from a He-Ne laser $(632.8 \mathrm{~nm}, 10 \mathrm{~mW})$ was coupled into the chip using direct focusing with an objective lens (10x, NA 0.25) and was collected upon exiting by another objective (40x, NA 0.65 ) before being directed to a silicon photodetector (PD, Hamamatsu S1337-33BR) connected to an acquisition system for signal readout and for further signal analysis. Light from another He-Ne laser was focused by a lens with a focal distance of 75 $\mathrm{mm}$ on the cantilever. A spot with diameter of about $30 \mu \mathrm{m}$ was positioned at the cantilever end. The reflected beam was projected on to a two-sectional position sensitive photodetector (PSD) to monitor the displacement of the cantilever. 


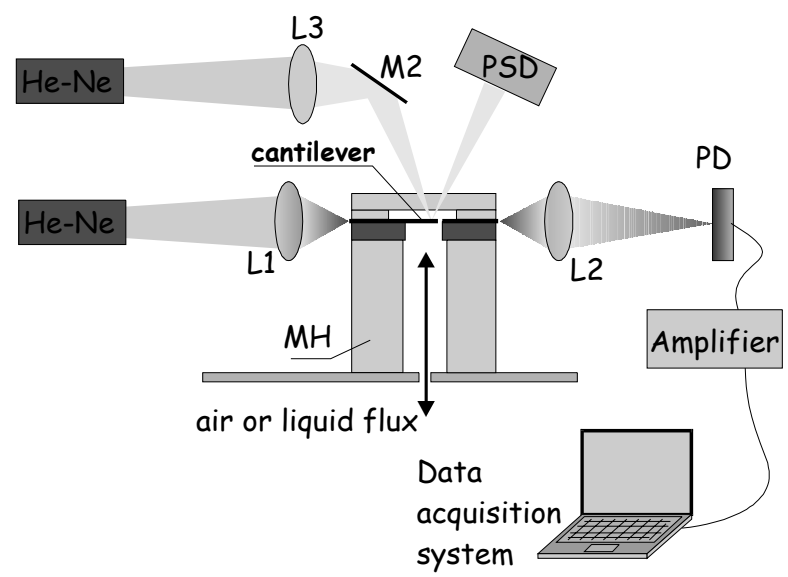

Fig. 3. The experimental setup used for characterization of the sensor. $L 1, L 2$ are objective lenses, $L 3$ is a plano-convex lens, $P S D$ is position sensitive photodetector, $M$ is a mirror, $P D$ is a conventional photodetector, $M H$ is a microfluidic header.

The optical cantilevers can be operated either in a static or in a dynamic mode. In the absence of external forces the cantilever deformation can be only provoked by the mechanical stress gradient within the cantilever [2], this property is employed in the biological sensors detecting superficial stress on the cantilevers. Cantilevers with an adsorbed molecular layer or metallised cantilevers represent at least bi-layer structures. The presence of analyte species may produce heating due to adsorption of them onto the cantilever surface or due to the heating produced by the subsequent chemical reaction. In case of thin cantilevers mechanical stresses also can be affected directly by the adsorption without heating. Thus, for example, the adsorption of the molecules on the atomically pure crystalline faces induces significant surface stress changes. The stress gradient may be induced or changed also directly by temperature changes making the cantilever sensors suitable for microcalorimetry applications, already demonstrated on silicon nitride cantilevers covered with gold [21]. Stress gradient can not be produced in the uniform optical cantilevers. The static deflections of optical waveguide cantilevers not covered by absorptive material were demonstrated exerting an air pressure on the cantilevers (see Fig. 4).

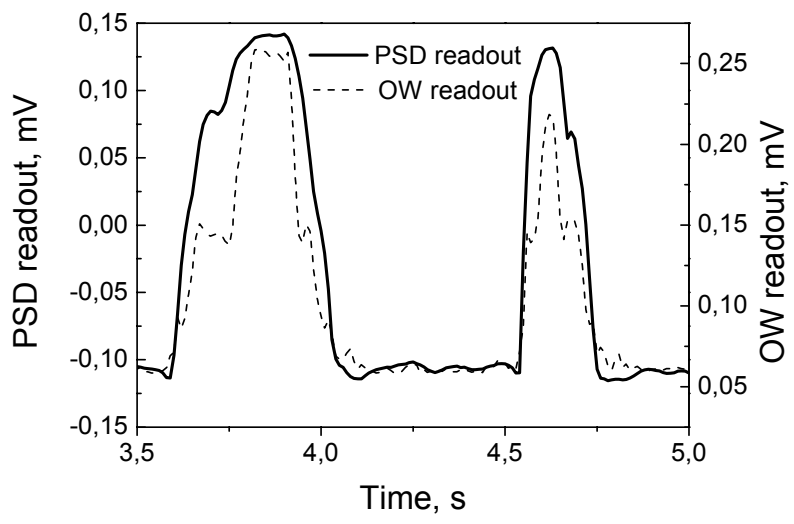

Fig. 4. Static deflection induced by exerting an air flux on the cantilever. The cantilever deflection was measured using both the optical lever and butt-coupled waveguide techniques. The signal change by $100 \mathrm{mV}$ corresponds to the cantilever free end displacement by about $1 \mu \mathrm{m}$ for both methods.

In the resonant mode, rather suitable for vacuum and gas environment applications, the cantilever itself is a mechanical resonator. A spectrum of thermo-mechanical noise of a cantilever registered 
using the proposed method is presented in Fig. 5. The intrinsic vibration of the cantilever produced by the particles, like Brownian motion of small particles in liquids, allows for operation of the cantilevers either with external excitation or without it. The changes in the resonant frequencies of the cantilevers can be induced by the adsorbate-induced mass loading, changes in the spring constant due to the chemical reaction in the cantilever, or due to viscosity changes in liquid medium. In dynamic mode it is easier to distinguish the signal from noise. But operation in liquids is accompanied by low quality factor and this does not make this mode more sensitive in case of low frequency cantilever resonators.

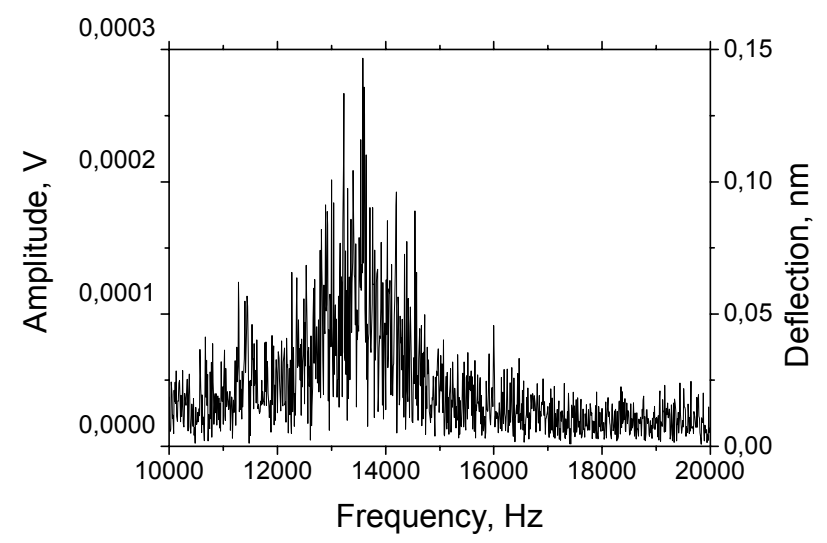

Fig. 5. Thermo-mechanical noise of the optical waveguide cantilever registered using the proposed method [20].

\section{OWC FABRICATION TECHNOLOGY}

The cantilevers were fabricated from silicon dioxide following the technology which schematic view is presented in Fig. 6. The silicon dioxide cantilevers are perfectly transparent and demonstrate low stress gradient if the bottom layer of a few hundred nanometres is eliminated.

The technology was started with $300-\mu$ m-thick silicon $<100>$ wafers, polished on both sides. Thermal silicon dioxide layer with thickness of $1.0 \mu \mathrm{m}$ was grown by wet oxidation of the wafer at $1100{ }^{\circ} \mathrm{C}$. PECVD silicon dioxide layer with thickness of $1.5 \mu \mathrm{m}$ was deposited on the back side of the wafer. Then $180 \mathrm{~nm}$ thick silicon nitride layer was deposited using LPCVD technique on both sides. The silicon dioxide layer on the back side was used later as a mask material for etching the cavities by deep reactive ion etching (DRIE). Two photolithographic steps followed by reactive ion etching were applied to the components side, first to define the waveguides in silicon nitride and second to define the cantilevers. The silicon nitride beside the waveguides was etched down to 40 $\mathrm{nm}$, so that thin $\mathrm{Si}_{3} \mathrm{~N}_{4}$ film covered the cantilevers. Cavities under the cantilevers were defined by standard photolithography and dry etching on the back side. DRIE was applied from both the components side and from the back side. Double side DRIE was carried out to remove $5 \mu \mathrm{m}$ and 290 $\mu \mathrm{m}$ of silicon from the components side and from the back side respectively. This was done in order to maintain the dimensions of the cavities on the components side with precision of 1-2 $\mu \mathrm{m}$, what would be impossible due to the undercut if only DRIE from the back side was applied. The rests of the silicon under the cantilevers were removed by wet etching in TMAH solution $\left(25 \% \mathrm{w}\right.$. at $\left.80^{\circ} \mathrm{C}\right)$. Then the cantilevers were thinned to $600 \mathrm{~nm}$ from the bottom by wet etching in SiO-etch solution. Finally, $40 \mathrm{~nm}$ of silicon nitride were removed in hot orthophosphoric acid $\left(80 \%\right.$ at $\left.160^{\circ} \mathrm{C}\right)$. The technology allows for near $100 \%$ yield. 


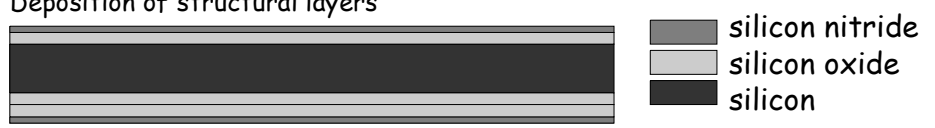

Structural layer patterning,

Definition of the cantilevers and

the cavities under the cantilevers

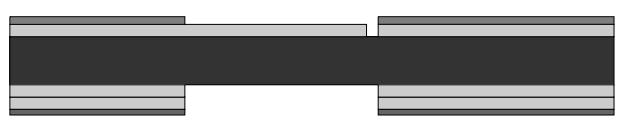

DRIE from both structural and back sides

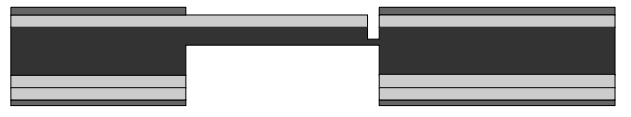

TMAH etching of silicon under the cantilevers Wet etching of the cantilevers from the bottom

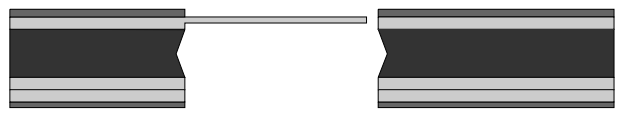

Fig. 6. Technology o fabrication of the OWC chip.

The external facets of the input and output waveguides were made extended by a couple of microns over the border of the chip. This helped to avoid waveguide facets polishing and to provide relatively efficient coupling using direct focusing and to collect light exiting the receptor using the full numerical aperture. The facets formed by conventional reactive ion etching allowed to obtain coupling efficiency comparable with the one achieved on polished waveguides.

\section{METALLISED OWC}

The technology of immobilisation or the technology of stress generation in the cantilever is dependent generally on the kind of reaction to detect. There are various mechanisms producing reaction and changes in Gibbs free energy associated with adsorption process. One of them is chemisorption of molecules on gold coated cantilever [2]. The self-assembly thiol-based chemistry is probably the most common in the experiments on bimolecular interactions [1, 3-7, 21-23].

Use of gold layer on optical cantilevers is challenging, but not impossible. First of all absorptive gold layer on the cantilever surface causes high losses. On the other hand, gold may induce initial bending of the cantilevers, and the free ends should be accurately aligned, or it is better to say accurately misaligned by a certain distance [20], with the output waveguide and this technological problem should be properly addressed while fabricating gold coated optical waveguide cantilevers. Depending on the cantilever geometry and gold layer thickness light might be only partially absorbed. In Fig. 7 there are presented the absorption losses for the structure composed of silicon oxide waveguide covered with gold surrounded by air as a function of gold layer and the cantilever thickness. 


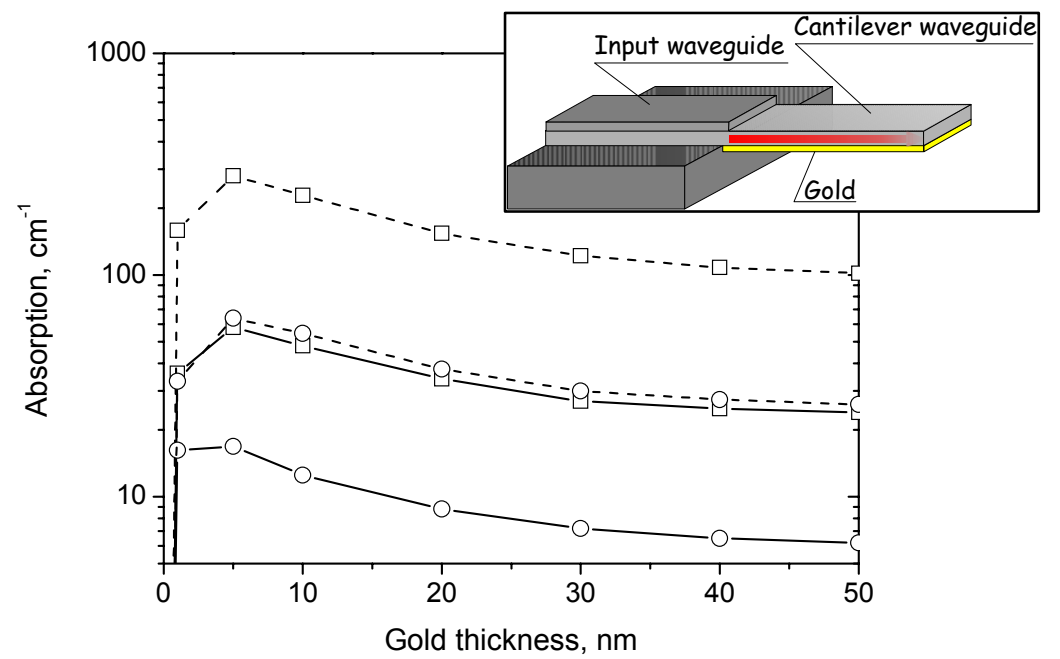

Fig. 7. Absorption loss in the metallic layer versus the thickness of the layer and the waveguide cantilever thickness simulated using the transfer matrix approach. Solid line is for the fundamental mode, dash line is for the first mode. Squares: cantilever is $600 \mathrm{~nm}$ thick, circles: cantilever is $1000 \mathrm{~nm}$ thick. In the inset: a schematic view of a cantilever with gold layer at the bottom.

Absorption of the first mode is much stronger if compared with absorption of the fundamental mode. This can be addressed to relatively high concentration of the electromagnetic field in vicinity of the gold layer for the first mode. The single mode operation of the cantilever is desirable [20], so filtering the high order modes by applying an absorptive layer is helpful if the cantilever is a multimode waveguide. To make an experiment a thin (about $5 \mathrm{~nm}$ ) gold layer was deposited on the bottom surface of the multimode cantilevers. The cantilever profiles before and after metallisation are shown in Fig. 8. The cantilever profile has been changed after deposition due to the stress gradient created by the absorptive layer.

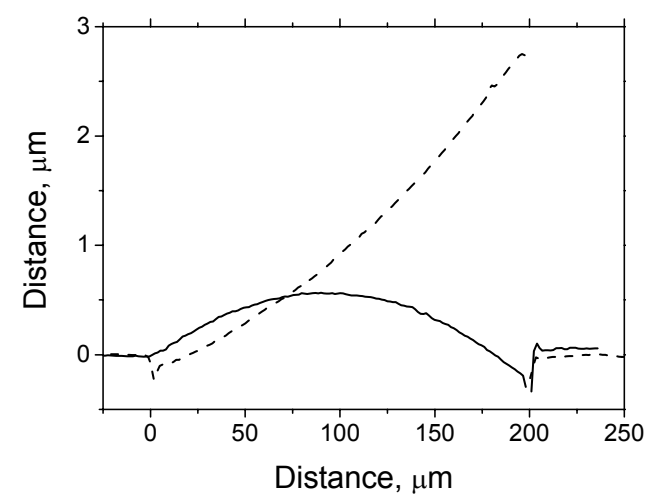

Fig. 8. The cantilever profiles before (dash line) and after (solid line) metallisation. The cantilever initially deflected upward was bent down by the mechanical stress produced by the bimetallic effect. 
The distribution of intensity of light exiting the cantilever was measured using the setup shown in Fig. 3 and actuating the cantilever by air flux. The power of light exiting the output waveguide was correlated against the cantilever deflection. The distribution is presented in Fig. 9.

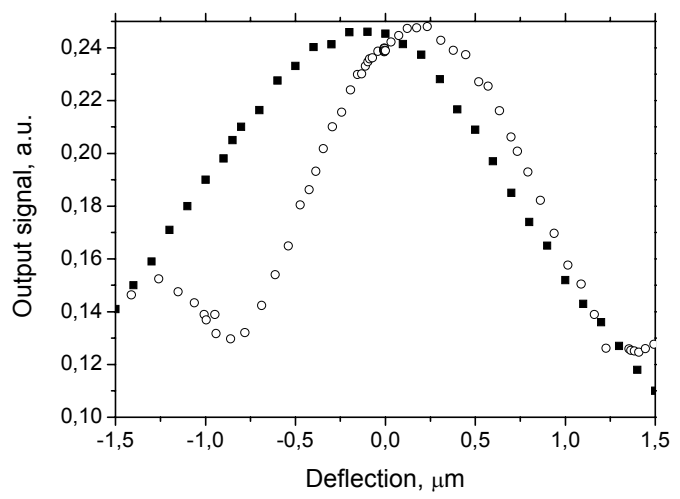

Fig. 9. Distribution of light intensity at the cantilever exit before (circles) and after (squares) metallisation. After metallisation the fundamental mode only is observed in the distribution.

The cantilever with gold absorptive layer is a bimetallic structure which is able to bend with the temperature changes. Introducing light into the cantilever results in absorption of the electromagnetic energy and its transformation in heat basically in the gold layer. The expansion of the gold layer results in deflection of the cantilever. This phenomena can be employed to place the cantilever free end to the required position changing the power of light coupled into the cantilever. In Fig. 10 we present a time diagram of a cantilever actuated by light. Light was modulated by a mechanical chopper, cantilever deflection monitored by optical lever method. The peaks correspond to the moments when the light was on. $100 \mathrm{mV}$ on the PSD correspond to $1 \mu \mathrm{m}$ displacement of the cantilever end. The power of light coupled into the cantilever was around $0.5 \mathrm{~mW}$

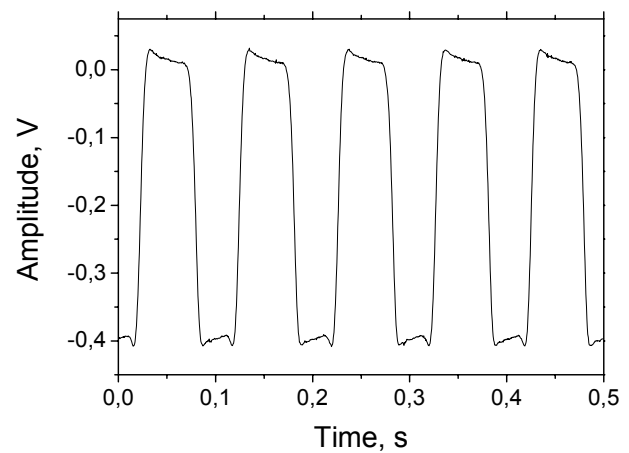

Fig. 10. Cantilever deflection due to absorption of light in the gold layer. The time diagram of the signal obtained from the PSD detector (see Fig. 3). The signal was the difference between the voltages generated in the photodetector sections. $100 \mathrm{mV}$ voltage corresponds to the cantilever free end displacement of $100 \mathrm{~nm}$.

It can be observed on the graph that each peak has a fast but finite rise time and then a slight exponential decrease which was addressed to the heat transfer into the silicon oxide layer of the cantilever. This process of heat dissipation is relatively slow and subject to saturation. The actuation of the cantilever by light was carried out up to frequencies of $1000 \mathrm{~Hz}$, when under the same conditions the amplitude of cantilever deflection went down by a factor of two. 


\section{LIGHT COUPLING AND CHIP ENCAPSULATION}

\subsection{Light Coupling}

Planar topology of the sensor helps for easy integration of the device on a lab-on-chip. For successful use of the device the problems of light coupling and encapsulation should be properly addressed. The sensitivity of the device is generally defined by the output light power related to the shot noise of the photodetector, by the cantilever thermo-mechanical noise, and by stability of light coupling into the input waveguide.

The coupling can be provided either by end-fire technique, or by excitation of the waveguides on the diffraction gratings. Preliminary experiments [20] were done using coupling by means of direct focusing with an objective lens. For efficient coupling the light beam should be focused to a submicron spot size. The tolerances for efficient coupling are counted then in nanometers to provide stability needed for acceptable detection limit of the sensor. Even with fine micropositioning system these tolerances are difficult to maintain. The output signal is floating in long term and sensitive to minute vibrations. Focusing in several channels is even more challenging using this method.

Light coupling to several channels can be realised by means of beam splitters integrated on the chip, light focused to an input waveguide can be distributed among the channels.

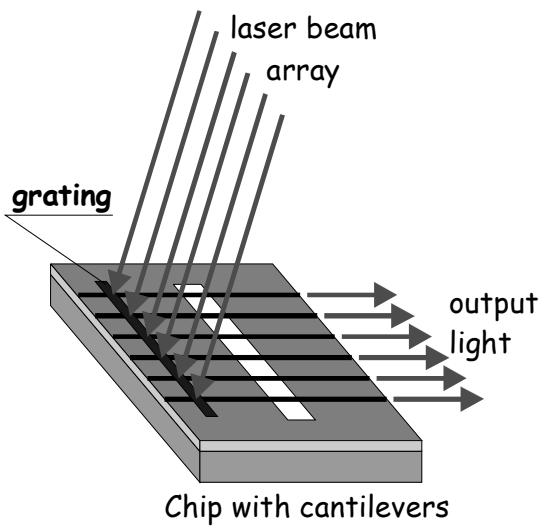

Fig. 11. Light coupling into waveguide array on the diffraction grating. Light from an array of laser sources is focused by an optical system (not shown here) onto the diffraction grating covering all the waveguides.

Excitation on the diffraction grating is probably the easiest way of stable light coupling in several channels simultaneously. The grating can be fabricated to cover each channel and excitation may be realised using an array of laser sources and a relatively simple optical system consisting of cylindrical lenses (see Fig. 11). The stability of the diffraction grating coupling potentially is better than the direct focusing. The appropriate parameters of the corrugation should be applied. One of the disadvantages of the diffraction grating excitation method is a complexity of the technology of fabrication of the devices. Another drawback is a regress to the out-of-plane topology of the setup (like in the case with optical lever readout), which lowers the overall system integration level.

The schematics of experiments using the optical cantilever transducers is presented in the block diagram in Fig. 12. 


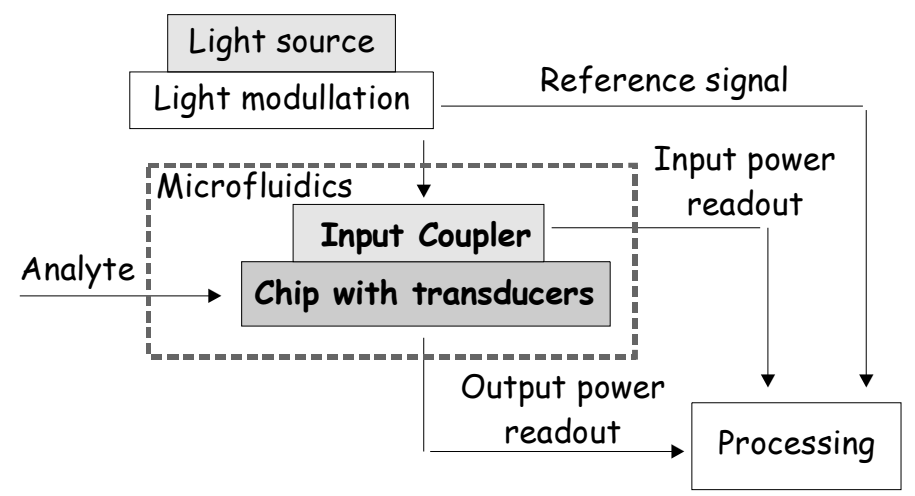

Fig. 12. Block diagram of the experimental setup with optical waveguide cantilever transducer. Input coupler (can be waveguide facet or diffraction grating) is integrated on the chip. Input power is controlled during the experiment, so that changes in the output signal could be properly addressed. Light modulation can be realised mechanically (mechanical chopper) or electronically (modulating LED current). Reference signal from light modulator is used for synchronous detection.

The waveguide channel with the cantilever is a linear system. If the optical parameters of the channel are maintained constant then the power at the exit is linearly proportional to the power coupled at the input, unless the changes due to the cantilever deflection are introduced. Thus the problem of instability of light source and coupling efficiency can be solved by providing the monitoring of the coupled power. In the present embodiment of the sensor chip the coupled power can be monitored at the IWG/Cantilever junction, where light partially escapes from the channel. Light modulation and synchronous detection are essential to protect the system from the ambient light and to abstract the information about the cantilever motion from the overall signal changes.

\subsection{Encapsulation}

The microfluidic header share some basic requirements including the need to integrate the microfabricated cantilever arrays with a flow cell enabling reagents to be introduced to the cantilevers in a reproducible way, and subsequently removed to waste. In reported experiments the duration of the reaction can last from a half an hour [6] to several hours [23]. The time, among other factors, depends on the volume of the microfluidics cell where the chip with cantilevers is mounted. The liquid volume in the header should be minimised and the height of the liquid above the cantilevers should be no more than about $200 \mu \mathrm{m}$ in order to reduce diffusion times in the sample. The integrated microfluidic header and cantilever array should form the disposable part of the system, in order to avoid contamination issues between experiments. This assembly therefore needs to be relatively inexpensive. All materials used in the microfluidic header must also be biocompatible. For the common path microfluidic header, a single flow path is required which addresses all of the cantilevers in the array. It is also desirable to minimise the number of transitions between materials of different refractive indices. Unless the diffraction grating is employed, the direct focusing method is used for coupling. The chip is accessible for both coupling of light using direct focusing and for monitoring the cantilevers deflections using optical lever technique. It is assumed then that both input and output waveguide facets will be accessible and the lid of the cell is polished and optically transparent. Acrylic (PMMA) was chosen as the structural material for the microfluidic headers as it meets the above requirements and also is readily machinable. Another useful property of acrylic is that multiple layers may be stacked together and then bonded.

There has been considered a number of flow configurations for the microfluidic header. Figure 13 shows the configuration that was selected for the microfluidic header with common flow path. This arrangement has two outlet ports. One outlet port is used for priming only, in order to ensure that all air bubbles can be removed from the header. Once the header has been primed with buffer solution 
and all air bubbles removed, the priming port is closed off and all flow is then directed parallel to the cavity.

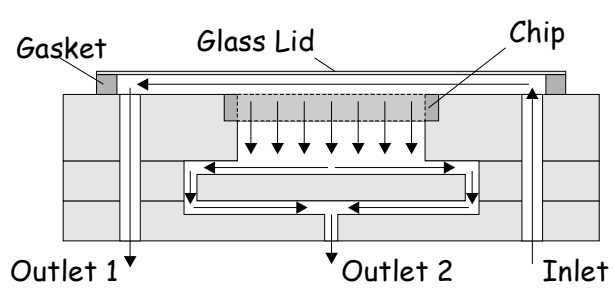

a)

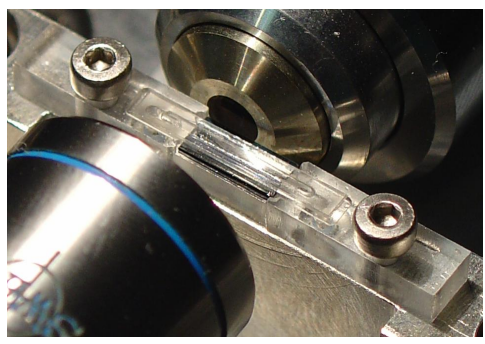

b)

Fig. 13. The schematic drawing of the microfluidics header used in the experiment.

The glass lid with thickness of $100 \mu \mathrm{m}$ used in the header is located close to the cantilevers so that there is a gap of about $200 \mu \mathrm{m}$ between the lid and the cantilevers. This allows to reduce the distortions of light beam focused onto the cantilevers, used for monitoring the cantilever deflections by optical lever readout. The overall volume of the cavity above the cantilevers is about $2.4 \mu$.

\section{CONCLUSIONS}

We presented a sensor based on the optical waveguide cantilever transducers. It was experimentally demonstrated that the cantilevers can bear a gold layer deposited on one of their surfaces. The gold layer can be used for the biomolecular recognition using thiol-based chemistry. Absorption losses are high, nevertheless the device can be operated if the power budget allows for it. Waveguide optical modes filtering is provided automatically by exploitation of the absorbing layer on the cantilever. This is helpful, since allows for predictable behaviour of the device. The experiments in air demonstrated that the cantilever with absorbent layer can be actuated by light, taking advantage of the power absorbed in this layer and the bi-metallic effect.

The optical cantilever device allows for high integration level. Some concepts related to the light coupling has been addressed and the methods for light coupling proposed. Taking into account the abilities of the sensor to work with absorbent layer on top it can be also concluded that besides to be operated in transparent environment the sensor can be operated in semitransparent environment as well. For the encapsulation of the chip an Acrylic (PMMA) microfluidic header with two outlet ports was manufactured.

\section{Acknowledgements}

The work has been done within the Optonanogen project supported by EC (IST-2001-37239) and NanoOptoChip project supported by CSIC (Spain) (2004-50F0300). Authors would like to thank to D. Wenn (iXscient Ltd) and N. Harris (University of Southampton) for the development of the microfluidic header.

\section{REFERENCES}

1. L. G. Carrascosa, M. Moreno, M. Alvarez, L. M. Lechuga, "Nanomechanical biosensors: a new sensing tool," Trends in Analytical Chemistry, Vol. 25, 196-206 (2006)

2. N. V. Lavrik, M. J. Sepaniak, P. G. Datskos, "Cantilever transducers as a platform for chemical and biological sensors," Rev. Sci. Instrum. vol. 75, 2229-2253 (2004)

3. K. M. Hansen, T. Thundat, "Microcantilever biosensors," Methods, vol. 37, 57-64, (2005)

4. Mechanics of Materials , S. Timoshenko, J. M. Gere, 1st edition, D. Van Nostrand Company, 1972

5. G.G. Stoney, Proc. Roy. Soc. Lond. Vol. A82, 172 (1909) 
6. Y. Arntz, J. D. Seelig, H. P. Lang, J. Zhang, P. Hunziker, J. P. Ramseyer, E. Meyer, M. Hegner and Ch. Gerber, "Label-free protein assay based on a nanomechanical cantilever array," Nanotechnology, vol. 14, 86-90 (2003)

7. N. Backmann, Ch. Zahnd, F. Huber, A. Bietsch, A. Pluckthun, H.-P. Lang, H.-J. Guntherodt, M. Hegner, and Ch. Gerber, "A label-free immunosensor array using singlechain antibody fragments," PNAS, vol. 102,14587-14592 (2005)

8. L.M. Lechuga, J.Tamayo, M. Álvarez, L.G. Carrascosa, A. Yufera, R. Doldán, E. Peralías, A. Rueda, J.A. Plaza, K. Zinoviev and C Domínguez, A. Zaballos, M. Moreno and C. Martínez-A, D. Wenn, N. Harris, C. Bringer, V. Bardinal, T. Camps, "A highly sensitive microsystem based on nanomechanical biosensors for Genomics applications," Sensors and Actuators B, Vol. 118, 2-10 (2006)

9. M. Yue, H. Lin, D. E. Dedrick, S. Satyanarayana, A. Majumdar, A. S. Bedekar, J. W. Jenkins, S. Sundaram, "A 2-D microcantilever array for multiplexed biomolecular analysis," J. Microelectromech. Syst., vol. 13, 290-299 (2004)

10. T. Fukuma, M. Kimura, K. Kobayashi, K. Matsushige, H. Yamada, "Development of low noise cantilever deflection sensor for multienvironment frequency-modulation atomic force microscopy," Rev. Sci. Instrum. vol. 76, 053704 (2005)

11. C. Schonenberger and S. F. Alvarado, "A differential interferometer for force microscopy," Rev. Sci. Instrum. vol. 60, 3131-3134 (1989)

12. J. Thaysen A. Boisen, O. Hansen, S. Bouwstra, "Atomic force microscopy probe with piezoresistive read-out and a highly symmetrical Wheatstone bridge arrangement," Sensors and actuators A: Physical, vol. 83, 47-53 (2000)

13. G. Shekhawat, S.-H. Tark, V. P. Dravid, "MOSFET-Embedded Microcantilevers for Measuring Deflection in Biomolecular Sensors," Science, vol. 311, 1592 (2006)

14. C. Kocabas, A. Aydinli, "Design and analysis of an integrated optical sensor for scanning force microscopies", IEEE Sensor J. vol. 5, 411-418 (2005)

15. G. G. Yaralioglu, A. Atatar, S. R. Manalis, and C. F. Quate, "Analysis and design of an interdigital cantilever as a displacement sensor," J. Appl. Phys., vol. 83, 7405-7415 (1998)

16. R. Budakian and S. J. Putterman, "Force detection using a fiber-optic cantilever," Appl. Phys. Lett. vol. 81, 2100-2102 (2002)

17. J. Mertens, M. Álvarez, and J. Tamayo, "Real-time profile of microcantilevers for sensing applications," Appl. Phys. Lett. Vol. 87, 234102 (2005)

18. S. Jeon and T. Thundat, "Instant curvature measurement for microcantilever sensors," Appl. Phys. Lett. Vol. 85, 1083 (2004)

19. M. Alvarez, J. Tamayo. "Optical sequential readout of microcantilever arrays for biological detection," Sensors and Actuators B, vol. 106, 687-690 (2005).

20. K. Zinoviev, C. Dominguez, J.A. Plaza, V. Cadarso L.M. Lechuga, "A novel optical waveguide microcantilever sensor for the detection of nanomechanical forces," $J$. Lightwave Technol., vol. 24 , 2132-2140 (2005)

21. S. L. Biswal, D. Raorane, A. Chaiken, H. Birecki, and A. Majumdar, "Nanomechanical Detection of DNA Melting on Microcantilever Surfaces," Anal. Chem. vol. 78, 7104-7109 (2006)

22. R. McKendry, J. Zhang, Y. Arntz, T. Strunz, M. Hegner, H.P. Lang, M. K. Baller, U. Certa, E. Meyer, H.-J. Güntherodt, and C. Gerber, "Multiple label free biodetection and 
quantitative DNA-binding assays on a nanomechanical cantilever array," PNAS, vol. 99, 9783-9788 (2002)

23. G. Wu, R. H. Datar, K. M. Hansen, T. Thundat, R. J. Cote, and A. Majumdar, "Bioassay of prostate-specific antigen (PSA) using microcantilevers," Nature Biotechnology, vol. 19, $856(2001)$ 